\title{
A new autogenous mobile system driven by vibration without impacts, excited by an impulse periodic force
}

\author{
The-Hung Duong ${ }^{1}$, Van-Du Nguyen ${ }^{2, *}$ and Ngoc-Tuan $\mathrm{La}^{3}$ \\ ${ }^{1}$ Department of Civil Engineering, Thai Nguyen University of Technology, Viet Nam \\ ${ }^{2}$ Department of Mechanical Engineering, Thai Nguyen University of Technology, Viet Nam \\ ${ }^{3}$ Department of Manufacturing Engineering, Vinh University of Technology Education, Viet Nam
}

\begin{abstract}
This report describes a new proposed design for autogenous mobile systems which can move without any external mechanisms such as legs or wheels. A Duffing oscillator with a cubic spring, which is excited by an impulse periodic force, is utilized to drive the whole system. The rectilinear motion of the system is performed employing the periodically oscillation of the internal mass interacting without collisions with the main body. Utilizing the nonlinear restoring force of the cubic spring, the system can move in desired directions. When the ratio between the excitation force and the friction force is smaller than 2.5 , backward or forward motion can be easily achieved by applying an excitation force in the same desired direction. Different from other vibro-impact drifting devices, no impact needed to drive the new proposed system. This novel structure allows to miniaturize the device as well as to simplify the control algorithm thus can significantly expand applicability of the proposed system.
\end{abstract}

\section{Introduction}

Mobile systems have been widely employed both in large- and micro-scale sizes. Such systems are preferable for special engineering applications in remote and hostile environments such as engineering diagnosis to disaster rescues or pipeline inspection [ $\underline{1}-\underline{3}$ ] and impact moling [는 $\underline{6}$. In micro-scale biomechanics, locomotion systems can enhance capabilities of the capsule endoscopy to deal with problems of traditional systems such as inability to control and stop, and risk of capsule retention [7]. Of such movable dynamical systems, vibration-driven platforms are a new kind which is propelled by the periodic motion of internal masses or the periodic deformation of their bodies. Using vibration-driven mechanism, several complications induced by external driving mechanism (wheels, chain tracks, or legs) can be avoided [ $\underline{8}, \underline{9}]$. The vibration-driven locomotion systems have been widely investigated from many aspects, including modeling and theoretical analysis and control [ $10-$ 13], design and experimental implementation [1417]. Several experimental platforms have been developed, employing centripetal forces generated by platform-mounted vibration micro-motors [14], electromagnetic force induced in voice coil motor $[15,16]$, centrifugal forces of a couple-pendulum [17]. The common issue of such vibration-driven systems is that, a complex control for the inertial mass motion is necessary to obtain the desired progression of the system $[10,15]$. Moreover, the problem for the large-scale mobile platforms also make difficult to miniaturize the platform. The capsule model using vibro-impact device proposed by Liu et al. [9] addressed these issues. A similar mobile mechanism in a large-scale platform of size which can produce strong impact force for moling machines was also reported [6]. Those models are inspired by a vibro-impact drifting oscillator studied by Pavlovskaia et al. [18]. Experimental verification [19-21] and dynamic response analysis of such systems [22-23] have been investigated in order to carry out the best performance conditions, including the system stability and maximum progression rate. For capsule endoscopy applications, the system abilities for moving forwards and backwards are required. In order to address this issue, a position feedback control method suitable to control the vibro-impact capsule system which uses a soft impact and harmonic excitation was proposed [24]. However, requirement of constraint setup in vibo-impact oscillator still make it difficult for minimizing the device for capsule applications. This paper propose a new design of a drifting actuator which can drive the autogenous mobile system without any impacts. The actuator is a kind of Duffing oscillator, as similar to the one proposed [25], containing a cubic spring but excited by impulse force. Our study revealed that by choosing proper values of design and operational parameters, the system can 
move in a desired direction with a simple control strategy. Nonlinear spring is a prerequisite condition for the movable ability of the system.

\section{Mathematical model}

A physical model of the proposed system is depicted in Figure 1, where a movable mass $m_{l}$ is connected with the system frame $m_{2}$ by a cubic spring $F_{s p r}$ and a viscous damper with damping coefficient $c$. The system is excited by a periodic impulse force $F$ with amplitude $A$ and frequency $\Omega$ exerts on both masses in opposite directions. The excitation amplitude, $A$ is assigned as a positive value if it acts on the mass $m_{l}$ in the forward direction and vice versa.

In this study, the excitation force is a $50 \%$ duty cycle force and thus can be expressed in a form of the Heaviside function as:

$$
\left\{\begin{array}{l}
F=\left\{\begin{array}{l}
A, \sin (\Omega t)>0 \\
0, \sin (\Omega t) \leq 0
\end{array}\right. \\
\text { or } F=\text { Heavisde }(\sin (\Omega t))
\end{array}\right.
$$

The friction force $F_{f}$ has direction opposite to the velocity of the frame body. $X_{1}$ and $X_{2}$ are the displacement of the inertial mass $m_{l}$ and the system body $m_{2}$, respectively.

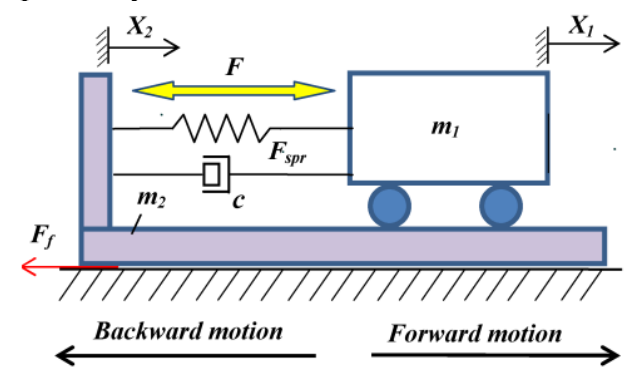

Fig. 1. Physical model of the system

The equations describing the motion of the system can be written as

$$
\left\{\begin{array}{l}
m_{1} \frac{d^{2} X_{1}}{d t^{2}}=F-F_{s p r}-c\left(\frac{d X_{1}}{d t}-\frac{d X_{2}}{d t}\right) \\
m_{2} \frac{d^{2} X_{2}}{d t^{2}}=-F+F_{s p r}+c\left(\frac{d X_{1}}{d t}-\frac{d X_{2}}{d t}\right)-F_{f} \\
F=\left\{\begin{array}{l}
A, \sin (\Omega t)>0 \\
0, \sin (\Omega t) \leq 0
\end{array}\right. \\
F_{f}=f \cdot\left(\mathrm{m}_{1}+\mathrm{m}_{2}\right) \cdot g \cdot \operatorname{sgn}\left(\frac{d X_{2}}{d t}\right)
\end{array}\right.
$$

where $d() / d t$ denotes differentiation with respect to time, $t ; \operatorname{sgn}()$ denotes the sign of velocity $\left(d X_{2} / d t\right) ; f$ is the friction coefficient between the frame body and the environment; $g$ is the gravitational acceleration.

An illustration of the $50 \%$ duty cycle forcing function with amplitude of $2 \mathrm{~N}$ and frequency of $5 \mathrm{~Hz}$ $(\Omega=31,416 \mathrm{rad} / \mathrm{s})$ is depicted in Fig. 2 .

The spring force, $\mathrm{F}_{\mathrm{spr}}$ is expressed as;

$$
F_{s p r}=k_{1}\left(X_{1}-X_{2}\right)+k_{2}\left(X_{1}-X_{2}\right)^{3}
$$

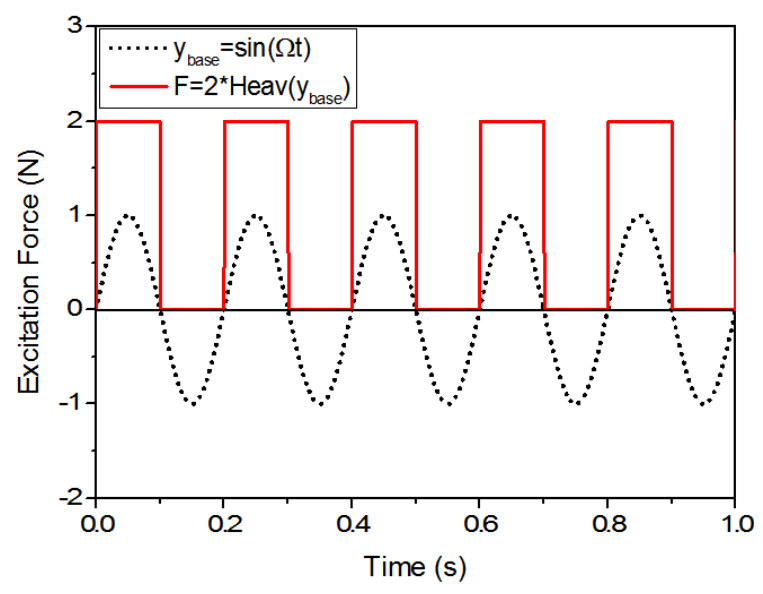

Fig. 2. Time history of the excitation force (red solid line) with frequency of $5 \mathrm{~Hz}$, and the corresponding sine function (black dots)

The Equations (1) can be rewritten in a dimensionless form as

$$
\left\{\begin{array}{l}
x_{1}^{\prime \prime}=\alpha H-\left(x_{1}-x_{2}\right)-\beta\left(x_{1}-x_{2}\right)^{3}-2 \zeta\left(x_{1}^{\prime}-x_{2}^{\prime}\right) \\
x_{2}^{\prime \prime}=\left[\begin{array}{l}
-\alpha H+\left(x_{1}-x_{2}\right)+\beta\left(x_{1}-x_{2}\right)^{3} \\
+2 \zeta\left(x_{1}^{\prime}-x_{2}^{\prime}\right)-\operatorname{sgn}\left(x_{2}^{\prime}\right)
\end{array}\right] / \mu
\end{array}\right.
$$

where

$$
\left\{\begin{array}{l}
\tau=\Omega_{0} t ; x_{1}=\frac{k_{1}}{F_{f}} X_{1} ; x_{2}=\frac{k_{1}}{F_{f}} X_{2} ; \Omega_{0}=\sqrt{\frac{k_{1}}{m_{1}}} ; \omega=\frac{\Omega}{\Omega_{0}} ; \\
\zeta=\frac{c}{2 m_{1} \Omega_{0}} ; \alpha=\frac{A}{F_{f}} ; \beta=\frac{k_{2}}{k_{1}}\left(\frac{F_{f}}{k_{1}}\right)^{2} ; \mu=\frac{m_{2}}{m_{1}} ; \\
H=h \operatorname{eav}(\sin (\omega t))
\end{array}\right.
$$

There are two response issues are usually considered for a mobile system: the progression rate and directional motion of the system. As can be seen, the Equations (5) contains five parameters which can be classified into two groups: $\beta$, $\zeta$ and $\mu$ can be considered as design parameters; $\alpha$ and $\omega$ are the parameters of operation task. Several effects of such parameters on the system response will be elicited in the following sections.

\section{Design parameters}

In this study, the progression rate, $P$, is considered as the displacement of the system frame, $x_{2}$ for the dimensionless time of 100 . The mass ratio is kept as constant $\mu=1$, e.g. the two masses have the same value. Firstly, the coupled effect of the stiffness, $\beta$ and the damping, $\zeta$ of the nonlinear spring on the progression rate, $x_{2}$ is considered. Figure 2 presents a contour plot of $x_{2}$ for $\beta \in[0,100]$ and $\zeta \in[0,1.0]$. All other parameters are fixed as $\mu=1, \alpha=1$ and $\omega=2$. 


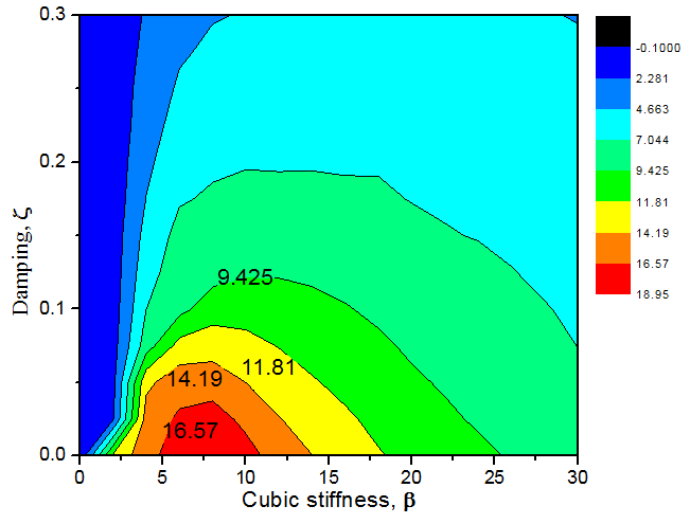

Fig. 3. (Color online) A contour plot of $P$ with respect to $\beta$ and $\zeta$; The values obtained for $\alpha=1, \omega=2$ and $\mu=1$

As can be seen on Figure 3, the progression rate reached values higher than 16.57 for $\beta \in[5,10]$ and $\zeta \in[0,0.3]$. Our numerical data revealed that, $P$ get peak value $P=17.571$ at $\beta=8$ and $\zeta=0.025$. Figure 3 depicts $x_{2}$ as a function of the cubic stiffness, $\beta$, for four different levels of the damping factor, $\zeta$ to support this remark.

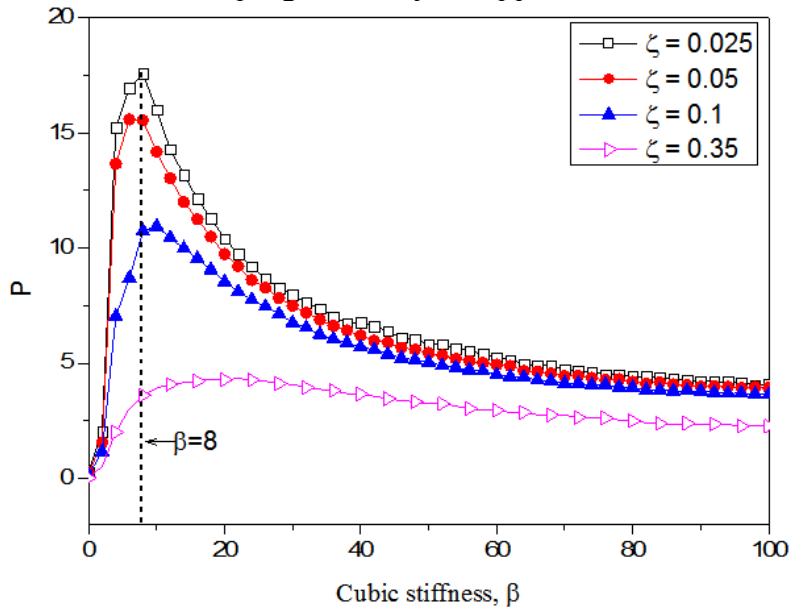

Fig. 4. A plot of $P$ as a function of $\beta$ for for four levels of $\zeta$; values obtained for $\alpha=1, \omega=2$ and $\mu=1$

As can be seen in Figure 4, the progression rate is significant when $\beta$ increases from 0 to 8 . It is worth to highlight that, the system is not able to move $(P=0)$ for $\beta=0$. However, the progression rate trends down when $\beta>8$. In other words, the spring inside the system must be a nonlinear spring in order to provide a movable ability. Besides, the cubic term, $\beta$, should be chosen carefully to obtain the fastest progression rate. It is also noted that there have three apexes of $x_{2}$ appeared remarkably around $\beta \approx 8$ for small damping values $(\zeta=0.025 ; \zeta=0.05$ and $\zeta=0.1$ ). For higher values of damping, such as $\zeta=0.35$ as shown in the Figure, changing $\beta$ would not have significant effect on the progression rate.

It is also observed from Figure 3 and Figure 4 that, the system with smaller damping values would provide higher progression rate. The next section presents some primary results obtained from system analysis under the effects of operational parameters.

\section{Operational parameters}

In order to understand the system behavior under different values of operational parameters, two design parameters are kept as constants as: $\mu=1, \zeta=0.025$. Figure 5 presents the bifurcation diagram of the progression rate, $P$, and the relative velocity, $v_{1}-v_{2}$, with respects to the excitation force, $\alpha$.

From Figure 5(a), it can be observed that the progression rate for positive $\alpha$ is exactly as the same as that for negative $\alpha$ but in opposite sign. The system can move with the highest progression rate for $|\alpha| \approx 1$. For $\alpha \in[-2.5,2.5]$, the system move forward when $\alpha>0$ and backward when $\alpha<0$. When increasing the force ratio, $|\alpha|>2.5$, the system moves back if $\alpha>0$ and vice versa, i.e. the system trends to move in opposite direction of the excitation force.

The bifurcation diagram of the relative velocity, $v_{l^{-}}$ $v_{2}$ shown in Figure 5(b) is a projection of the Poincaré map on the vertical axis. The calculations were run for 400 cycles of the excitation force. The first 100 cycles were taken to be transient and not included so as to plot the steady state of next 300 cycles.

(a)

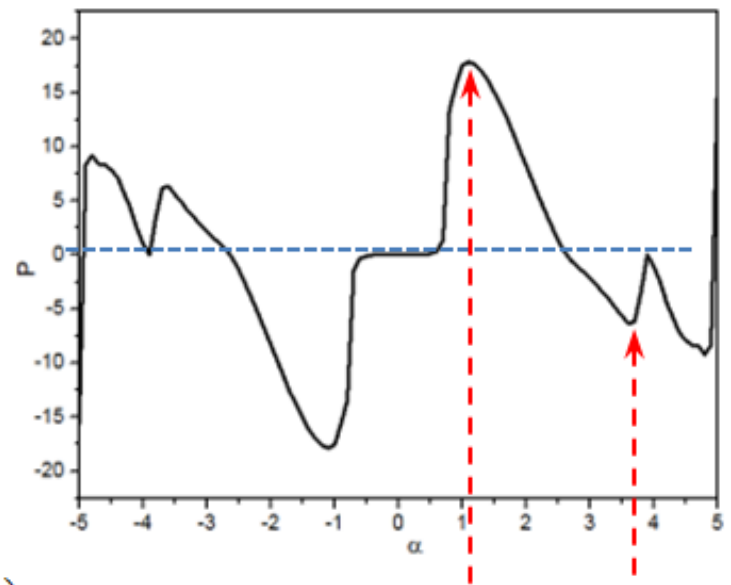

(b)

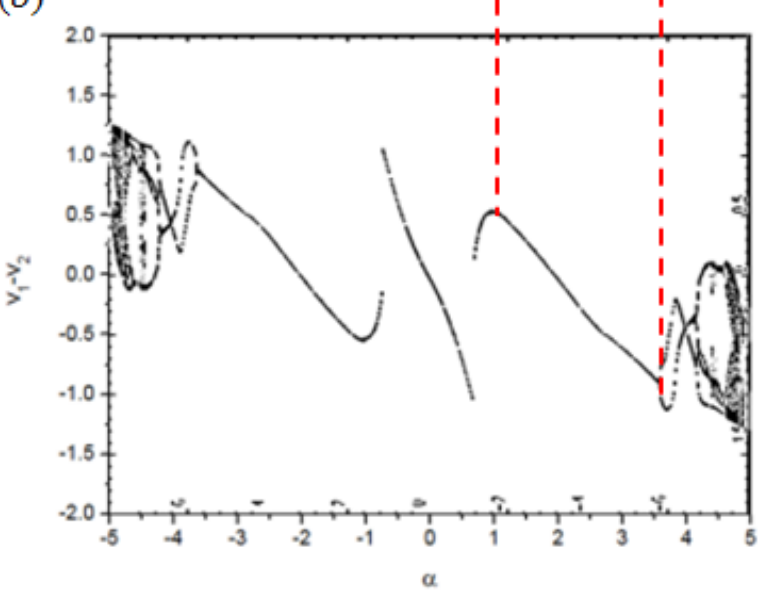

Fig. 5. Bifurcation diagram of (a) the progression rate, $P$ and (b) relative velocity $v 1-v 2$ with respects to the force ratio, $\alpha$.

The results obtained for $\mu=1, \zeta=0.025$ and $\omega=2.0$

As can be seen, the highest progression rate appeared when the mass motion has a period-one 
response. Besides, a peak of reverse movement (the progress movement is opposite to the excitation force)

(a)

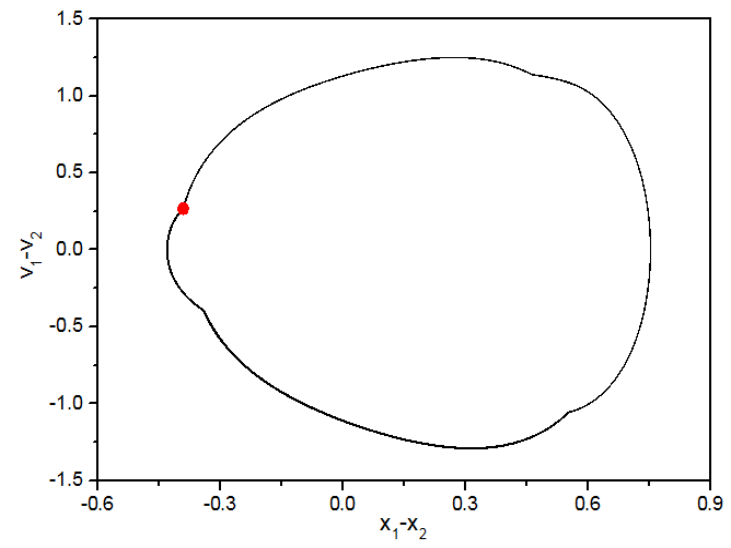

(b)

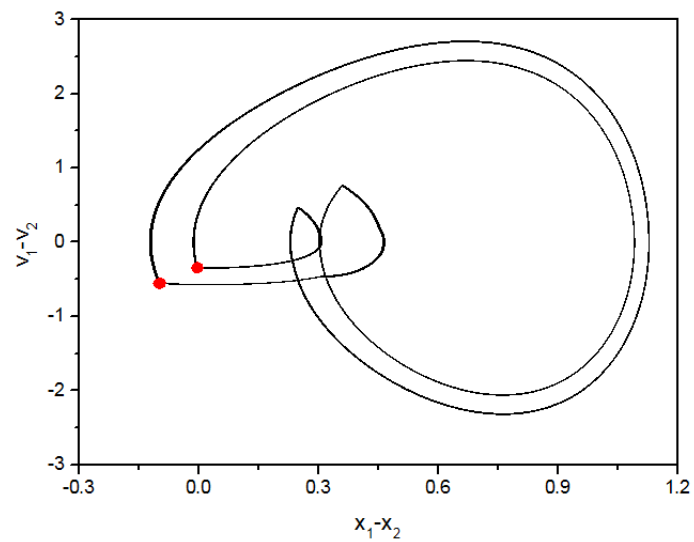

appeared where the relative motion is a period-two and higher. Figure 6 depicts these phenomena.
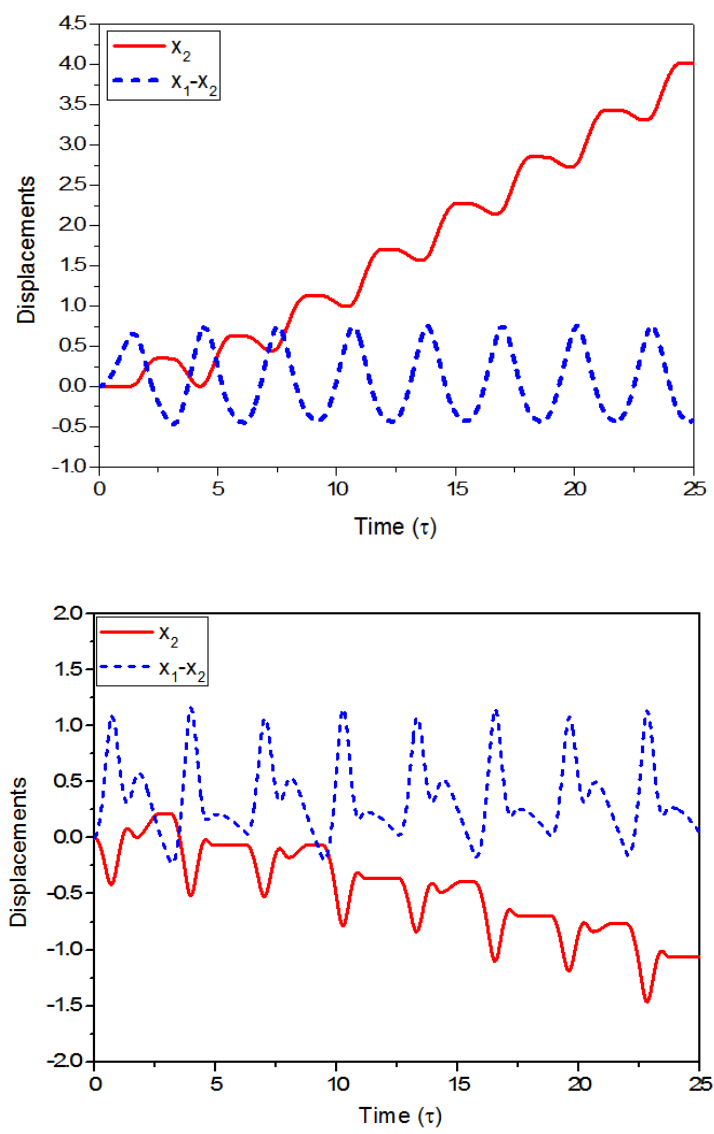

Fig. 6. (Color online) Left hand-side sub-plots: Trajectories presented by gray lines on the phase plane $\left(x_{1}-x_{2}, v_{1}-v_{2}\right)$, where Poincaré sections are marked by red dots; Right hand-side sub-plots: Time histories of the relative motion, $x_{1-x_{2}}$ (black dashed lines) and of the system displacement, $x_{2}$ (red solid lines). The calculations obtained for $\beta=8, \mu=1, \omega=2, \zeta=0.025$ and (a) $\alpha=1$, (b) $\alpha=3.75$

In Figure 6(a), the relative motion of the inertial mass is period-one, the system frame has a steady state movement in forward direction. When the mass has period-two response as shown in Figure 6(b), the system progressed in backward direction.

The influence of excitation frequency on the system progression is scrutinized by checking with the bifurcation diagram with respect to $\omega \in[0.1,5.1]$, as shown in Figure 7.

In overall, for all the values of the investigated excitation frequency, $\omega$, the system always progresses in forward direction, as the same as the direction of the excited force. There appears one significant local maximum of forward progression for $\omega=2$. For $\omega \in[1$, $2]$, increasing the excitation frequency would result in raising up the progression rate. However, for $\omega>2$, increasing the excitation frequency would reduce the progression rate. When the motion of the inertial mass change from period-one to period-two motion at $\omega=3.1$, the progression rate increase slightly. There appears a small peak of progression rate in the range of $\omega \in[3.1$, 3.75 ] but much smaller than the peak at $\omega=2$. (a)

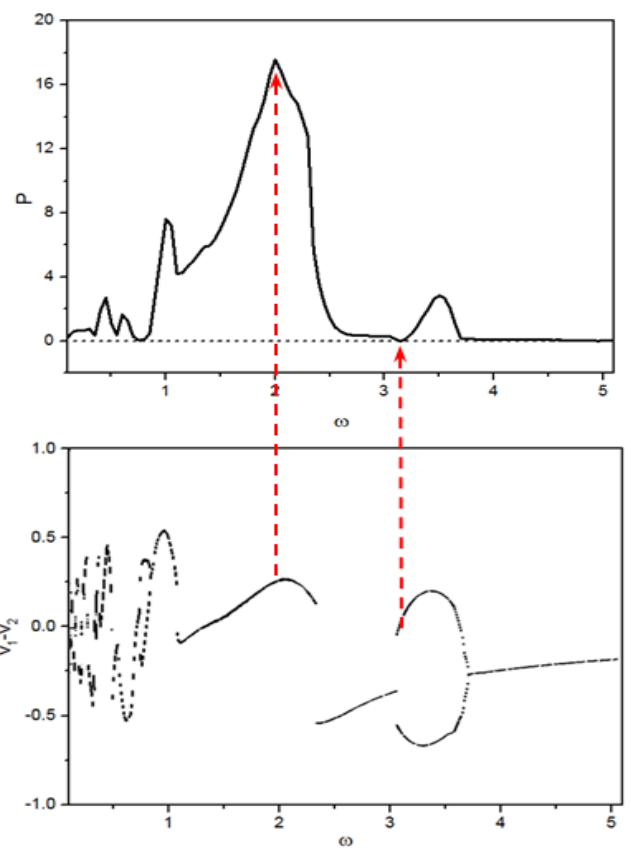

Fig. 7. Bifurcation diagram of (a) the progression rate, $P$, and (b) relative velocity $v_{1}-v_{2}$ with respects to the excitation frequency, $\omega$. The results obtained for $\mu=1, \zeta=0.025$ and $\alpha=1$ 


\section{Conclusion}

A new vibration-driven device which is able to drive an autogenous mobile system in desired directions has been developed. The most advantage of the new design is that the system can move in desired direction without impacts, thus can simplify the device structure. Moreover, the desired direction of the system movement can be obtained simply by applied an excitation force with the same direction as the desired motion.

The heart of the new system is a Duffing oscillator including a nonlinear spring with a cubic term of stiffness. For the investigated range of the parameters, our numerical study revealed that, the system can obtain the highest progression rate for the set of following parameters: cubic spring stiffness $\beta=8$, amplitude of the excitation force $\alpha=1$ and the excitation frequency $\omega=2$. For this set of parameters, the system not only reaches the highest progression rate, but also moves in the same direction as the direction of applied force.

The mathematical model with dimensionless parameters allows extending the results to both large- and microscale of different mobile systems. Further study should be paid to the system response for wider ranges of both design and operation parameters so as to optimize system structure and enhance its working effectiveness.

Acknowledgements: This research is funded by Vietnam National Foundation for Science and Technology Development (NAFOSTED) under grant number 107.01-2012.33.

\section{References}

[1]. Y. Zhang, S. Jiang, X. Zhang, X. Ruan, D. Guo, A variable-diameter capsule robot based on multiple wedge effects, IEEE/ASME Trans. Mechatron. 16 241-254, DOI: 10.1109/TMECH.2009.2039942;

[2]. A. Shukla and H. Karki, Application of robotics in onshore oil and gas industry - a review Part I, Robot. Auton. Syst. 75, 490-507, (2016) DOI:10.1016/j.robot.2015.09.012.

[3]. A. Shukla and H. Karki, Application of robotics in onshore oil and gas industry - a review Part II, Robot. Auton. Syst. 75, pp508-524, (2016) DOI:10.1016/j.robot.2015.09.013.

[4]. J. Warminski, S. Lenci, M.P. Cartmell, G. Rega, M. Wiercigroch, Nonlinear Dynamic Phenomena in Mechanics, Vol 181; Chapter 5:Engineering Applications of Non-smooth Dyn., Springer, pp. 211273, (2012) DOI:10.1007/978-94-007-2473-0_5;

[5]. E. Pavlovskaia, M. Wiercigroch, K.C. Woo and A.A. Rodger, Modelling of Ground Moling Dynamics by an Impact Oscillator with a Frictional Slider, Meccanica 38 (1), pp85-97, (2003), DOI:10.1023/A:1022023502199;

[6]. V.D. Nguyen, K.C. Woo and E. Pavlovskaia, Experimental study and Mathematical Modelling of a New of Vibro-impact moling device, Int. J. Nonlinear. Mech. 43 (6), pp542-550, (2008) DOI:10.1016/j.ijnonlinmec.2007.10.003;

[7]. L. Liu, S. Towfighian and A. Hila, A review of locomotion systems for capsule endoscopy, IEEE
Reviews in Biomedical Engineering (2015), DOI: 10.1109/RBME.2015.2451031;

[8]. N. A. Sobolev and K. S. Sorokin, Experimental Investigation of a Model of a Vibration-Driven Robot with Rotating Masses, J. Comput. Sys. Sc. Int. 46(5), pp826-835, 10.1134/S1064230707050140;

(2007); DOI:

[9]. Y. Liu, M. Wiercigroch, E. Pavlovskaia and H. Yu, Modeling of a vibro-impact capsule system, Int. J. Mech. Sci., 66, 2-11 (2013), DOI:10.1016/j.ijmecsci.2012.09.012;

[10]. H. Li, K. Furuta and F. L. Chernous'ko, Motion Generation of the Capsubot Using Internal Force and Static Friction; Proceedings of the 45th IEEE Conference on Decision \& Control, pp6575-6580, (2006) DOI: 10.1109/CDC.2006.377472;

[11]. F.L. Chernous'ko, The optimal periodic motions of a two-mass system in a resistant medium, J. Appl. Math. Mech. 72, pp 116-125, (2008) DOI:10.1016/j.jappmathmech.2008.04.014.

[12]. N.N. Bolotnik, T. Figurina, Optimal control of the rectilinear motion of a rigid body on a rough plane by means of the motion of two internal masses, J. Appl. Math. Mech. 72, pp126-135, (2008) DOI:10.1016/j.jappmathmech.2008.04.013;

[13]. N.N. Bolotnik, F.L. Chernous'ko, T. Figurina, Optimal Control of a Two-body Vibration-driven Locomotion System in a Resistive Environment, IFACPapersOnLine 48-25 (2015) 091-096, DOI:10.1016/j.ifacol.2015.11.065;

[14]. P. Vartholomeos and E. Papadopoulos, Dynamics, Design and Simulation of a Novel Microrobotic Platform Employing Vibration Microactuators, J. Dyn. Sys., Meas., Control 128(1), 122-133 (2005) DOI:10.1115/1.2168472;

[15]. G. Su, C. Zhang C, R. Tan and H. Li, A Design of the Electromagnetic Driver for the "Internal ForceStatic Friction" Capsubot, International Conference on Intelligent Robots and Systems, , (IEEE/RSJ 2009), DOI: 10.1109/IROS.2009.5354587;

[16]. M.N. Huda, H.N. Yu and S.O. Wane, Self-contained Capsubot Propulsion Mechanism, International Journal of Automation and Computing, 8(3), 348-356, (2011), DOI: 10.1007/s11633-011-0591-3;

[17]. Y. Liu, H. Yu and S. Cang, Modelling and motion control of a double-pendulum driven cart, Proc. IMechE Vol. 226 Part I: J. Systems and Control Engineering, 226 No. 2 175-187, (2012), DOI: 10.1177/0959651811414507;

[18]. E. Pavlovkskaia, M. Wiercigroch and C. Grebogi, Modelling of an impact system with a drift, Phys. Rev. E, 64, 056224 (2001), DOI: 10.1103/PhysRevE.64.056224;

[19]. V.D. Nguyen and K.C. Woo, New electrovibroimpact system, Proc. IMechE, Part C: J. Mech. Eng. SC., Vol. 222, (4), 629-642 (2008), DOI: 10.1243/09544062JMES833;

[20]. V.D. Nguyen and K.C. Woo, Nonlinear dynamic responses of new electro-vibroimpact system, $J$. Sound. Vib.; 310(4-5), pp. $769-775$ (2008), DOI: 10.1016/j.jsv.2007.10.032;

[21]. Y. Liu, E. Pavlovskaia and M. Wiercigroch, Experimental verification of the vibro-impact capsule model, Nonlinear Dyn., 83(1), 1029-1041 (2016), DOI: $10.1007 / s 11071-015-2385-6$; 
[22]. J.H. Ho, V.D. Nguyen and K.C. Woo, Nonlinear dynamics of a new electro-vibroimpact system; Nonlinear Dyn., 63(1-2), 35-49 (2011), DOI: 10.1007/s11071-010-9783-6;

[23]. Y. Liu, S. Islam, E. Pavlovskaia and M. Wiercigroch, Optimization of the Vibro-Impact Capsule System, Strojniški vestnik - Journal of Mechanical Engineering 62, 7-8, 430-439 (2016), DOI: 10.5545/svjme.2016.3754;
[24]. Y. Liu, E. Pavlovskaia, M. Wiercigroch, Z. Peng, Forward and backward motion control of a vibroimpact capsule system, Int. J. Nonlinear. Mech. 70, 30-46, (2015) DOI: 10.1016/j.ijnonlinmec.2014.10.009;

[25]. V-D. Nguyen, T-H. Duong, N-H. Chu and Q-H. Ngo, The effect of inertial mass and excitation frequency on a Duffing vibro-impact drifting system, Int. J. Mech. Sci., 124-125, pp. 9-21 (2017), DOI: 10.1016/j.ijmecsci.2017.02.023. 